\title{
Diffraction Quality Optimization and Data Collection at Ambient Temperatures under Humidity Controlled Conditions
}

\author{
Silvia Russia, Jeney Wiermanª, Aina Cohena, representing the entire SMB Team \\ a Stanford Synchrotron Radiation Light Source, 2575 Sand Hill Rd., Menlo Park 94025, California, \\ USA, srussi@slac.stanford.edu, jwierman@slac.stanford.edu, acohen@slac.stanford.edu
}

The Structural Molecular Biology (SMB) group of the Stanford Synchrotron Radiation Lightsource (SSRL) is developing a program for remote access crystallography data collection at near physiological temperatures and controlled humidity. The utility of controlled hydration to change the properties of protein crystals is well documented, beginning with the pioneering dehydrated hemoglobin and myoglobin studies of Perutz, Kendrew and Huxley (1940's-1950's). Crystal dehydration increases the protein/solvent ratio and may trigger changes in crystal packing, unit cell dimensions and space group which can change the internal order of the crystal lattice (mosaicity, diffraction power) and crystal/cryo-solution dynamics. Integrating controlled hydration within the pipeline of room temperature data collection will enable optimization per protein crystal sample. This capability also opens the door to new investigations on protein dynamics. To this end, a novel plate has been developed for crystallization, sample transport and automated sample mounting for diffraction data collection at ambient temperatures. Future automation at SSRL-SMB and LCLS-MFX will support diffraction data collection and crystal screening at ambient temperatures or $100 \mathrm{~K}$ using a rapid nozzle switcher. 